\title{
Estimation of the size of the iatrogenic Creutzfeldt-Jakob disease outbreak associated with cadaveric dura mater grafts in Korea
}

\author{
Byoung-Hak Jeon $^{1 *}$, Jinseob Kim², Ganghyun Kim³ ${ }^{3}$, Soochul Park ${ }^{4}$, SangYun Kim ${ }^{5}$, Hae-Kwan Cheong ${ }^{1}$ \\ ${ }^{1}$ Department of Social and Preventive Medicine, Sungkyunkwan University School of Medicine, Suwon; '2Department of Preventive Medicine, \\ Graduate School of Public Health, Seoul National University, Seoul; ${ }^{3}$ Department of Neurosurgery, National Medical Center, Seoul; ${ }^{4}$ Department \\ of Neurology, Yonsei University of College of Medicine, Seoul; ${ }^{5}$ Department of Neurology, Seoul National University Bundang Hospital, \\ Seongnam, Korea
}

OBJECTIVES: This study estimated the overall incidence of iatrogenic Creutzfeldt-Jakob disease (iCJD) based on dura graft cases in Korea using a mathematical model.

METHODS: We estimated the number of annual dura grafts performed between 1980 and 1995 by applying the proportion of dura grafts recorded by the Health Insurance Review Agency claim dataset in Korea to the number of nationwide neurosurgery cases. The distribution of the incubation period was assumed to fall under a Weibull distribution with density function or a log-logistic distribution with density function.

RESULTS: The total number of neurosurgery procedures performed from 1980 to 1995 was estimated to be 263,945, and among those operations, 37\% used dura graft products. Between the years of 1980 and 2020, our model predicted that the total number of iCJD cases would be between 14.9 and 33.2 (95\% confidence interval [CI], 13.4 to 50.9). Notably, we estimated that the cumulative number of iCJD cases caused by dura grafts between 1980 and 2011 was approximately 13.3 to 27.3 (95\% CI, 12.2 to 40.6).

CONCLUSIONS: Based on our model, we postulate that the incidence of iCJD will sharply decline from 2012 to 2020. However, additional new cases are still expected, which necessitates a strong national surveillance system.

KEY WORDS: Prion diseases, Creutzfeldt-Jakob disease, Dura mater, Modeling, Surveillance, Incubation period, Prediction

\section{INTRODUCTION}

Creutzfeldt-Jakob disease (CJD) is a rare, devastating, chronic disease affecting the central nervous system. Widespread degradation of cells occurs in response to the pathogenic transformations caused by prion proteins in the central nervous system.

Correspondence: Hae-Kwan Cheong

Department of Social and Preventive Medicine, Sungkyunkwan University

School of Medicine, 2066 Seobu-ro, Jangan-gu, Suwon 16419, Korea

Tel: +82-31-299-6300, Fax: +82-31-299-6299, E-mail: hkcheong@skku.edu

${ }^{*}$ Current affiliation: Division of Public Health Preparedness and Response, Korea Centers for Disease Control and Prevention, Cheongju, Korea.

Received: Jul 26, 2016, Accepted: Dec 19, 2016, Published: Dec 19, 2016 This article is available from: http://e-epih.org/

(C) 2016, Korean Society of Epidemiology

(C) This is an open-access article distributed under the terms of the Creative Commons Attribution License (http://creativecommons.org/licenses/by/4.0/), which permits unrestricted use, distribution, and reproduction in any medium, provided the original work is properly cited.
CJD is peculiar in that it can develop either via a spontaneous degenerative process, as a result of genetic predisposition, or through transmission of the pathogenic prion protein [1]. Iatrogenic CJD (iCJD) is a transmitted form of CJD resulting from the healthcare-associated inoculation of infected human tissue in a victim. The incidence of CJD is between 0.5 and 2.0 per million globally and is quite homogenous across various regions [2,3]. Notably, iCJD accounts for less than $5 \%$ of the total number of prion diseases $[4,5]$.

The first case of iCJD was caused by corneal transplantation in 1974 [6]. Since this case, reports have been published of iCJD arising from the use of contaminated instruments or the inoculation of contaminated biogenic material, such as dura mater and hormones (human growth hormone or human gonadotropin-releasing hormone) extracted from cadavers $[7,8]$. There have been 469 total reports of iCJD worldwide, 236 of which were due to surgical procedures, including 228 cases from dura grafts, four cases from surgical instruments, two cases from em- 
bedded electroencephalography (EEG) needles, and two cases from corneal transplantation. Medical procedures were responsible for 233 cases, including 226 cases resulting from the use of growth hormone, four cases resulting from the use of gonadotropin-releasing hormone, and three cases arising from packed red blood cells donated from patients with latent CJD [8].

It was discovered that Lyodura grafts, made from human cadavers by a German company (B. Braun Melsungen AG, Melsungen, Germany), were a source of iCJD. In 1987, the first case of iCJD associated with cadaveric dura mater transplantation was reported in the US $[9,10]$. From then to 2012, 228 cases of iCJD associated with Lyodura grafts have been reported globally. Among these cases, 142 (62\%) occurred in Japan [8]. The most recent case was reported in Korea in 2011 [11].

The incubation period of dura graft-associated iCJD ranges from 1.3 to 30.0 years (mean, 12.0 years) [8]. Dura mater originating from cadavers was widely used in neurosurgery until the World Health Organization (WHO) recommended banning its use in 1997 [12]. Since then, reports of iCJD have declined markedly. With this reduction, it is expected that most cases of dura mater-associated iCJD will occur within a decade [13].

In Korea, a national CJD surveillance system has been operated by the Korea Centers for Disease Control and Prevention (KCDC) since 2001. The first case of iCJD in Korea was not reported until 2011, but the case was a patient who had received a dura graft 23 years earlier [11]. Because dura grafting was a popular surgical procedure, and because Koreans have a similar genetic background to Japanese, increasing concerns have been raised about the further development of iCJD cases in Korea. Therefore, it became necessary to estimate the risk of iCJD in the Korean population and to make predictions about future expectations. This study aimed to assess the risk of iCJD in the Korean population and to estimate the overall magnitude of the iCJD epidemic from dura grafts in Korea using a mathematical model. We validated this model by comparing our predictions to the actual number of cases detected through surveillance.

\section{MATERIALS AND METHODS}

\section{Estimation of the at-risk population for iatrogenic Creutzfeldt-Jakob disease}

To estimate the size of the population exposed to dura grafts, annual neurosurgery reports from all neurosurgery training hospitals in Korea from 1980 to 1995 were collected. Neurosurgery training hospitals are obligated to report all neurosurgical procedures to the Korean Neurosurgical Society annually as a process of quality control for residency training. The reports include an account of each operation, according to the Interna- tional Classification of Diseases (ICD), 9th revision, Clinical Modification (until 1995, ICD-10 afterward). From the annual reports, the annual number of all types of neurosurgery procedures and treatments and the number of residents and neurosurgeons were extracted. A total of 450 annual reports from 79 institutions for the years 1980 through 1995 were collected. Years with missing reports were imputed using a linear regression estimation based on the number of neurosurgeons and training doctors.

The Health Insurance Review Agency (HIRA) claims dataset in Korea for the years 2007 to 2011 was used to estimate the proportion of patients who had undergone dura grafting for each neurosurgical procedure. The claims data for 52 types of neurosurgical operations, each of which had the potential for using dura grafts, were extracted from the main HIRA claim database over the 5 -year period. Claims data included medications and procedure codes. All claims containing at least one of the 36 material codes associated with dura-containing material were extracted for each neurosurgical procedure. The probability of using dura grafts was estimated for each operation by dividing the number of neurosurgical claims containing the dura graft material by the number of total claims for each neurosurgical procedure. We then estimated the number of annual dura graft cases between 1980 and 1995 by summing the neurosurgery cases after calculating the probability of dura graft usage, which was estimated from the HIRA claim dataset (Figure 1).

\section{Duration of exposure scenario}

Two exposure scenarios were assessed. In scenario 1, the risk period was designated as 1980 to 1995 , with an equal level of risk across the period. In scenario 2, the period from 1980 to 1987 was set as a higher-risk period after consulting the Korean Neurosurgical Society on the patterns of dura grafting.

\section{Incubation period distribution}

To describe the distribution of the incubation period, the probability density function of the Weibull distribution and log-logistic distribution were used.The probability density function is widely applied to estimate the size of an epidemic [14,15].

For the probability density function of theWeibull distribution, we used the following equation:

$$
\mathrm{f}(\omega)=\lambda \gamma \omega^{\gamma-1} \exp \left(-\lambda \gamma \omega^{\gamma}\right)
$$

For the probability density function of the log-logistic distribution, we used the following equation:

$$
f(\omega)=\left(\lambda \gamma \omega^{\gamma-1} /\left(1+\lambda \omega^{\gamma}\right)^{2}\right.
$$

In these equations, $\mathrm{f}(\omega)$ represents the probability density function of the incubation period, $\omega$ is the reported incubation period, and $\gamma$ and $\lambda$ are unknown parameters. We estimated the un- 


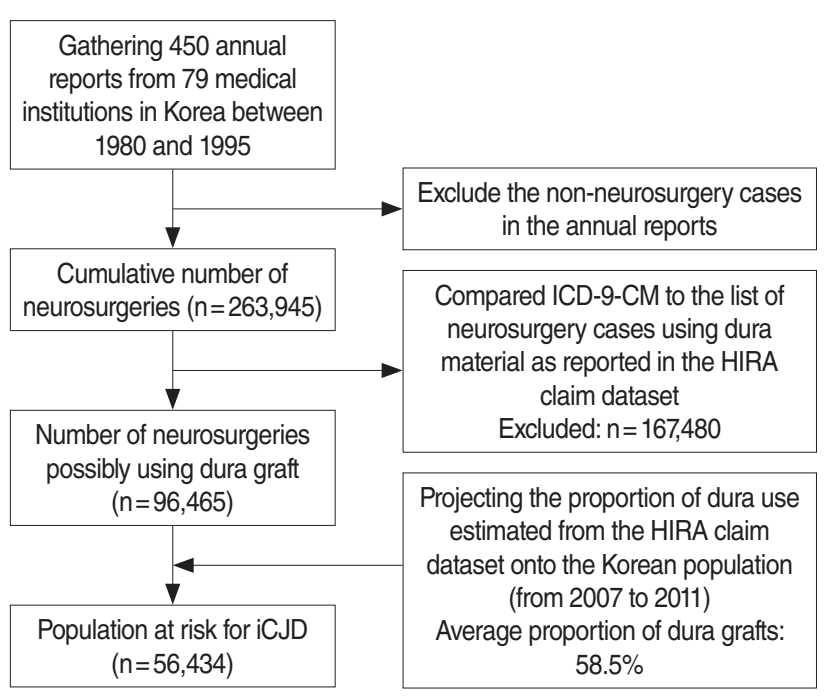

Figure 1. Process by which the dura graft population in Korea between 1980 and 1995 was estimated. ICD-9-CM, International Classification of Diseases, 9th revision, Clinical Modification; HIRA, Health Insurance Review Agency; iCJD, iatrogenic Creutzfeldt-Jakob disease.

known parameters $(\gamma, \lambda)$ to be the maximum likelihood function and to be greater than 0 [16-18], and we assumed $\omega$ to be between 1.3 and 30 years [18].

\section{Competing risk}

The competing risk was defined as the mortality rate of patients who underwent dura grafting. Four probabilities of competing risk in our analyses were considered: $\mathrm{z}=0, \mathrm{z}=0.02, \mathrm{z}=$ 0.05 , and $\mathrm{z}=0.1[18]$.

\section{Modeling the retrospective number of cases}

To model the total number of cases, the exposure population was defined by identifying iCJD risk factors in the dura graft neurosurgery cases between 1980 and 1995 (Table 1). The expected number of cases in each year was estimated. The key indicators were the population exposed to the iCJD risk factors $(\mathrm{N})$, the cumulative incidence of iCJD (p), the incubation period $(\omega)$, and the mortality rate of patients with a dura graft $(\mathrm{z})$.

To estimate the size of the iCJD outbreak, we used two models. One model applied a full scale $(\mathrm{p}=0.04 \%$ for the overall period, $\mathrm{p}=0.08 \%$ for the higher-risk period ) based on the incidence of the iCJD outbreak in Japan, and the other model used a half scale of the incidence $(p=0.02 \%$ for the overall period; $p=0.04 \%$ for the higher-risk period) $[8,19-21]$, where $f(\omega)$ is the probability density function of the incubation period $\left(\int_{0}^{\infty} f(\omega) d \omega\right), F(\omega)$ is the cumulative distribution function of the incubation period $\left(\int_{0}^{\omega} \mathrm{f}(\mathrm{k})\right.$ $\mathrm{dk}$ ), and $\mathrm{N}(\mathrm{t})$ is the cumulative number of $\mathrm{iCJD}$ cases at each specific time point $(t)[13,18,19]$, which is depicted as follows:
Table 1. Estimation of the population at risk for iatrogenic CreutzfeldtJakob disease from dura grafting in Korea from 1980 to 1995

\begin{tabular}{lrrrr}
\hline Year & $\begin{array}{r}\text { Neurosur- } \\
\text { geons (n) }\end{array}$ & $\begin{array}{r}\text { No. of total } \\
\text { neurosurgical } \\
\text { procedures }\end{array}$ & $\begin{array}{c}\text { No. of neuro- } \\
\text { surgical pro- } \\
\text { potential use } \\
\text { of dura grafts }\end{array}$ & $\begin{array}{c}\text { Estimated } \\
\text { number of } \\
\text { patients } \\
\text { exposed to } \\
\text { dura grafts }\end{array}$ \\
\hline 1980 & 75 & 6,920 & 1,880 & 1,100 \\
1981 & 92 & 9,076 & 2,724 & 1,594 \\
1982 & 103 & 11,239 & 3,087 & 1,806 \\
1983 & 114 & 11,434 & 3,947 & 2,309 \\
1984 & 130 & 12,811 & 4,345 & 2,542 \\
1985 & 143 & 13,620 & 4,433 & 2,594 \\
1986 & 146 & 14,635 & 4,499 & 2,632 \\
1987 & 155 & 15,194 & 4,923 & 2,880 \\
1988 & 160 & 16,850 & 5,896 & 3,449 \\
1989 & 177 & 17,287 & 6,286 & 3,677 \\
1990 & 184 & 17,723 & 6,463 & 3,781 \\
1991 & 195 & 20,672 & 7,871 & 4,604 \\
1992 & 199 & 21,784 & 8,266 & 4,835 \\
1993 & 211 & 23,483 & 9,237 & 5,404 \\
1994 & 244 & 24,058 & 9,840 & 5,757 \\
1995 & 273 & 27,159 & 12,768 & 7,470 \\
Cumulative total & & 263,945 & 96,465 & 56,434 \\
Annual average (1980-1995) & 16,497 & 6,029 & 3,527 \\
\hline & & & &
\end{tabular}

$$
\mathrm{N}(\mathrm{t})=\sum_{x=1980}^{2020} N \times p \times z \times F(t-x)
$$

The remaining parameters in the projection models were then estimated. Robustness against violation of the assumptions was confirmed by examining 16 combinations (Table 2) [13].

\section{Assessment of the actual number of iatrogenic Creutzfeldt-Jakob disease cases}

All cases between 1990 and 2011 were reviewed, and the cases suspicious for iCJD were selected based on the clinical findings and the risk factors identified in the case review format and medical records. Medical records and surveillance information sheets of the individual cases were reviewed by a neurologist (DWL) and an epidemiologist (SHK), who assigned a final consensus diagnostic categorization of each case as definite, probable, possible, or unlikely. In this study, the diagnostic criteria for accidentally transmitted transmissible spongiform encephalopathies developed by the National CJD Research and Surveillance Unit (NCJDRSU) were adopted [20].

An iCJD case was defined as a patient compatible with the diagnostic criteria of sporadic CJD as defined by NCJDRSU [20] with a history of one of the following exposures: (1) treatment with human pituitary growth hormone, human pituitary gonadotrophin, or a human dura mater graft, (2) a corneal graft in which the corneal donor was classified as having definite or 
Table 2. Estimation of the cumulative number of iatrogenic Creutzfeldt-Jakob disease cases associated with dura grafts in Korea according to different models and scenarios

\begin{tabular}{|c|c|c|c|c|c|c|c|c|c|c|c|}
\hline \multirow[b]{2}{*}{ Model $^{1}$} & \multirow{2}{*}{$\begin{array}{l}\text { Exposure } \\
\text { scenario }^{2}\end{array}$} & \multirow[b]{2}{*}{$P(\%)^{3}$} & \multirow{2}{*}{$\begin{array}{l}\text { Competing } \\
\text { risk }\end{array}$} & \multicolumn{4}{|c|}{ Weibull distribution ${ }^{4}$} & \multicolumn{4}{|c|}{ Log-logistic distribution 4} \\
\hline & & & & $\leq 2011$ & 2012-2020 & Total & $\begin{array}{c}\text { Incidence } \\
(\%)^{5}\end{array}$ & $\leq 2011$ & $2012-2020$ & Total & $\begin{array}{c}\text { Incidence } \\
(\%)^{5}\end{array}$ \\
\hline \multirow[t]{11}{*}{ Model 1} & 1 & 0.04 & 0 & 24.5 & 2.4 & 26.9 & 0.05 & 26.8 & 6.8 & 33.6 & 0.06 \\
\hline & & & 2 & 24.7 & 2.6 & 27.3 & 0.05 & 26.4 & 6.2 & 32.6 & 0.06 \\
\hline & & & 5 & 24.5 & 2.7 & 27.3 & 0.05 & 26.0 & 5.4 & 31.3 & 0.06 \\
\hline & & & 10 & 24.5 & 3.0 & 27.5 & 0.05 & 26.0 & 4.6 & 30.6 & 0.05 \\
\hline & 2 & 0.08 & 0 & 44.8 & 4.4 & 49.3 & 0.09 & 40.6 & 10.3 & 50.9 & 0.09 \\
\hline & & & 2 & 40.3 & 4.3 & 44.6 & 0.08 & 36.3 & 8.5 & 44.8 & 0.08 \\
\hline & & & 5 & 34.6 & 3.8 & 38.4 & 0.07 & 31.7 & 6.5 & 38.2 & 0.07 \\
\hline & & & 10 & 28.5 & 3.5 & 32.0 & 0.06 & 27.9 & 4.9 & 32.8 & 0.06 \\
\hline & Median & & & 26.6 & 3.2 & 29.7 & 0.05 & 27.3 & 6.3 & 33.2 & 0.06 \\
\hline & Minimum & & & 24.5 & 2.4 & 26.9 & 0.05 & 26.0 & 4.6 & 30.6 & 0.05 \\
\hline & Maximum & & & 44.8 & 4.4 & 49.3 & 0.09 & 40.6 & 10.3 & 50.9 & 0.09 \\
\hline \multirow[t]{11}{*}{ Model 2} & 1 & 0.02 & 0 & 12.2 & 1.2 & 13.4 & 0.02 & 13.4 & 3.4 & 16.8 & 0.03 \\
\hline & & & 2 & 12.3 & 1.3 & 13.7 & 0.02 & 13.2 & 3.1 & 16.3 & 0.03 \\
\hline & & & 5 & 12.3 & 1.4 & 13.6 & 0.02 & 13.0 & 2.7 & 15.7 & 0.03 \\
\hline & & & 10 & 12.3 & 1.5 & 13.8 & 0.02 & 13.0 & 2.3 & 15.3 & 0.03 \\
\hline & 2 & 0.04 & 0 & 22.4 & 2.2 & 24.6 & 0.04 & 20.3 & 5.2 & 25.5 & 0.05 \\
\hline & & & 2 & 20.1 & 2.2 & 22.3 & 0.04 & 18.2 & 4.2 & 22.4 & 0.04 \\
\hline & & & 5 & 17.3 & 1.9 & 19.2 & 0.03 & 15.8 & 3.3 & 19.1 & 0.03 \\
\hline & & & 10 & 14.2 & 1.7 & 16.0 & 0.03 & 13.9 & 2.5 & 16.4 & 0.03 \\
\hline & Median & & & 13.3 & 1.6 & 14.9 & 0.03 & 13.7 & 3.2 & 16.6 & 0.03 \\
\hline & Minimum & & & 12.2 & 1.2 & 13.4 & 0.02 & 13.0 & 2.3 & 15.3 & 0.03 \\
\hline & Maximum & & & 22.4 & 2.2 & 24.6 & 0.04 & 20.3 & 5.2 & 25.5 & 0.05 \\
\hline
\end{tabular}

${ }^{1}$ Model 1 applied the same attack rate as was observed in Japan ( $p=0.04 \%$ and $0.08 \%$ ), and model 2 applied an attack rate of half that observed in Japan $(p=0.02 \%$ or $0.04 \%)$.

'Exposure scenario 1 assumed that the risk was the same over the entire period (1980-1995), and scenario 2 assumed that the risk was higher between 1980 and 1987.

${ }^{3}$ Cumulative incidence was based on the incidence of iatrogenic Creutzfeldt-Jakob disease in Japan [18].

${ }^{4}$ Probability density function: Weibull distribution, $f(y)=\lambda \gamma \omega^{r-1} \exp \left(-\lambda \omega^{\gamma}\right)$; log-logistic distribution, $f(y)=\lambda \gamma \omega^{\gamma-1} /\left(1+\lambda \omega^{\gamma}\right)^{2}$.

${ }^{5}$ The estimated incidence of iatrogenic Creutzfelt-Jakob disease was defined as the number of new cases occurring in the exposure population (19802020) divided by the number of persons exposed to dura grafts (1980-1995).

probable human prion disease, and (3) exposure to neurosurgical instruments previously used in a case of definite or probable human prion disease. In this study, the cases of definite or probable CJD were retrospectively reviewed for the presence of a history of exposure to a procedure associated with risk. If a risk factor of iCJD was present in their history, an epidemiologist performed an additional investigation of any exposure to neurosurgical dura grafting. The iCJD cases were categorized as definite or probable according to the level of diagnostic certainty based on these criteria. This study was approved by the institutional review board of Samsung Medical Center.

\section{RESULTS}

The total number of neurosurgical procedures between 1980 and 1995 was estimated as 263,945 . Among them, $37 \%$ were estimated to have involved grafting with dura products, and the proportion of the population that underwent neurosurgical procedures exposed to dura products via neurosurgery was $21 \%$. The average number of dura grafts per year from 1980 to 1995 was 3,527 (range, 1,100 to 7,470 ) (Table 1).

The estimated range of the cumulative number of iCJD cases associated with dura grafting was between 12.2 and 44.8 cases through 2011. In model 1, we assumed the incidence of iCJD in Korea to be the same as the incidence of iCJD in Japan. The cumulative incidence during the overall period and during the higher-risk period was 0.04 and $0.08 \%$, respectively. According to the Weibull distribution model, the median predicted cumulative number of iCJD cases through 2011 was estimated to be 26.6 (range, 24.5 to 44.8), and 3.2 additional cases (range, 2.4 to 4.4) of iCJD are expected to occur between 2012 and 2020. According to the log-logistic model, the median cumulative number of iCJD cases through 2011 was estimated to be 27.3 (range, 26.0 to 40.6), and 6.3 additional cases (range, 4.6 to 10.3) of iCJD are expected to develop between 2012 and 2020 (Table 2). 


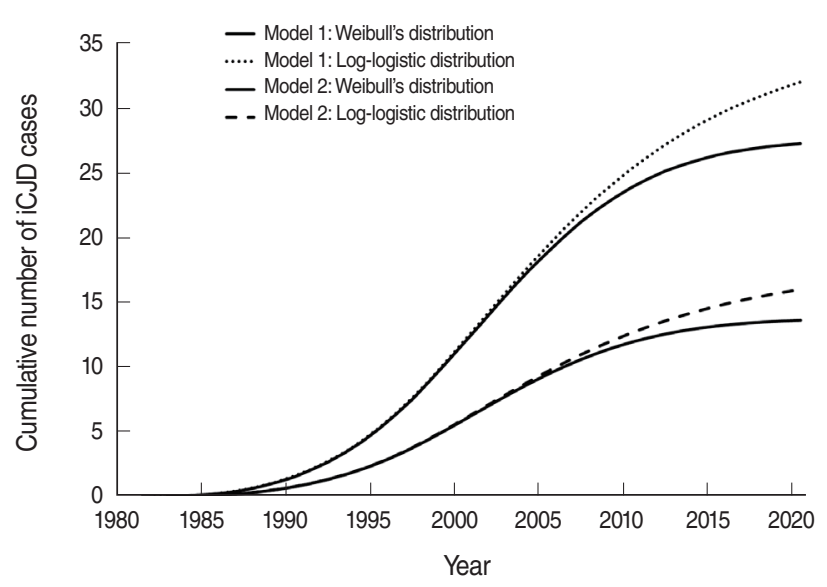

Figure 2. Projection of the cumulative number of iatrogenic Creutzfeldt-Jakob disease (iCJD) cases from 1980 to 2020 in Korea using the Weibull and log-logistic incubation period distribution for the overall exposure period (1980-1995). Model 1 set the attack rate to be the same as that observed in Japan ( $p=0.04 \%$ and $0.08 \%)$, and model 2 set the attack rate to be the half that seen in Japan ( $p=0.02 \%$ and $0.04 \%$ ).

In model 2, the incidence of iCJD was set to be half the incidence that was observed in Japan. The cumulative incidence in the overall period and the higher-risk period was calculated to be 0.02 and $0.04 \%$, respectively. In the Weibull model, the median cumulative number of iCJD cases through 2011 was estimated to be 13.3 (range, 12.2 to 22.4 ), and 1.6 additional cases (range, 1.2 to 2.2) are expected to occur between 2012 and 2020. In the log-logistic model, the median cumulative number of iCJD cases through 2011 was expected to be 13.7 (range, 13.0 to 20.3), and 3.2 additional cases (range, 2.3 to 5.2) of iCJD are expected to occur between 2012 and 2020 (Table 2).

We estimated the cumulative incidence of iCJD cases between 1980 and 2020 (Figure 2). Weibull and log-logistic models of distribution of the incubation period for the overall exposure period (1980 to 1995) were used. In our projective models, model 2 arrived at a lower estimate of the cumulative number of iCJD patients than model 1 , and the estimated number of cases, assuming a Weibull distribution, was lower than that predicted by the log-logistic distribution in both models.

Figure 3 shows the populations at risk for iCJD and the results of the projective models. In Korea, the exposure period for Lyodura was from 1980 to 1995 . Our model shows that the number of cases of iCJD increased continuously until 2001 and then decreased continuously thereafter, a trend that is expected to continue.

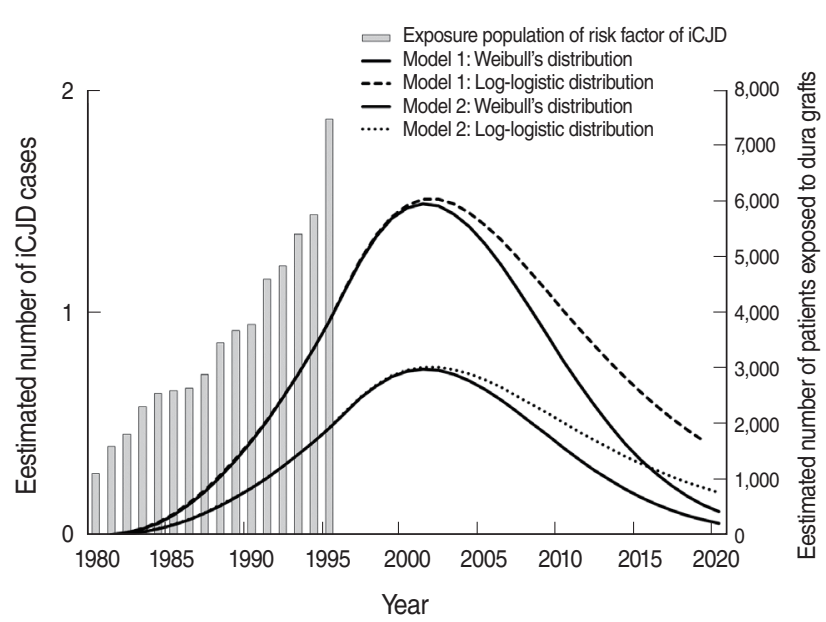

Figure 3. Estimated distribution of the incidence of iatrogenic Creutzfeldt-Jakob disease (iCJD) from 1980 to 2020 in Korea using the Weibull and log-logistic incubation period distributions for the overall exposure period (1980-1995). Model 1 set the attack rate at the same level as that observed in Japan ( $p=0.04 \%$ and $0.08 \%)$, and model 2 set the attack rate at half of the level as that observed Japan $(p=0.02 \%$ and $0.04 \%)$. The bar graph denotes the population at risk for iCJD.

\section{DISCUSSION}

The objectives of this study, which were to estimate the total size of the population at risk and the potential magnitude of the iCJD outbreak, were achieved, as 56,434 patients were estimated to have received dura grafts during the high-risk period between 1980 and 1995 (Table 1), and we estimated the size of the iCJD outbreak using all available data sources in Korea (Table 2). In our analysis, the at-risk population for iCJD was determined by extrapolating the present use rate of dura products from the German company (B. Braun Melsungen AG) in the HIRA claims data for the five years prior to this study because no information was available to estimate the rate of dura grafts in neurosurgery in the 1980s in Korea. In terms of the four typical distributions of predictive models, the estimated distributions of models 1 and 2 were similar. The number of cases estimated in the period (from 1980 to 2011) was similar to the actual number of iCJD cases reviewed by us.

It appears that the trend of our model was similar to that of the iCJD risk factor exposure population, with a time interval of 8 to 10 years between exposure to the dura graft and the estimated results. Based on these results, we are confident that our predictive models have high validity. This study demonstrated that the number of iCJD patients could reach a maximum of 10 persons in the 10 years following 2011. The range of the estimated incidence of $\mathrm{iCJD}$ was 0.02 to $0.09 \%$ between 1980 and 2020 (Table 2). The incidence of iCJD has gradually decreased. 
The results of the present study correspond to those associated with the major epidemic of dura mater-associated iCJD. In Japan, the mortality of iCJD was $0.06 \%$ (142 of 220,000 dura transplants) from 1979 to 2011 and $0.08 \%$ (81 of 100,000) during the high-risk period from 1983 to 1987 [8,21,22]. In Australia, the mortality rate of iCJD was $0.23 \%$ (5 of 2,208$)$ among patients who underwent dura grafting from 1979 to 2011 and $0.43 \%$ (5 of 1,172) for the high-risk period from 1982 to 1986 $[8,23]$. In France, 13 cases were linked to dura grafts from the mid-1980s to 1994, when the use of synthetic dura grafts was implemented. In 2005, the last French iCJD case due to a dura graft died, and no cases have since been reported in France [8,24]. Brown et al. $[8,25]$ suggested that the average incubation period for iCJD onset after Lyodura grafting is 12 years (range, 1.3 to 320.0 months), suggesting that the era of iCJD is approaching completion.

More than $60 \%$ (142 cases) of all patients (228 cases) with dura graft-associated CJD worldwide are from Japan [8,25], and the epidemiological data on iCJD caused by dura grafting in Japan $[22,26]$ have contributed to establishing the epidemiologic characteristics of iCJD. We referred to the prediction model and methods used in Japan and the UK [13,18,19], and we adopted the values of the model coefficients in our study. However, our estimation methods of the at-risk population were different than the methods reported by Tateishi [27]. Neurosurgeons in Japan estimated that the number of patients undergoing neurosurgical operations ranged from 100,000 to 120,000 annually, and that cadaveric dura was used in approximately one-fifth of these operations in the 1980s. Tateishi [27] estimated that cadaveric dura was used in about 20,000 patients per year, and Nakamura et al. [28] estimated that 260,000 patients received a cadaveric dura graft over the 13 years spanning from 1979 to 1991 . Although we adopted the prediction equation and the distribution of the reported incubation period of iCJD from former reports, the reason our models successfully predicted the actual cases is that the population at risk was estimated based on actual cases, and the risk exposure period was empirically derived from actual cases reported by neurosurgeons in Korea.

The incidence of iCJD patients has never been studied in Korea, although the KCDC has operated a national CJD surveillance system since 2001. This is the first Korean study that statistically estimated the magnitude of the iCJD outbreak using a predictive model. However, this study has important limitations, most of which stem from selecting indexes that were retrospectively calculated and reported. First, since we have no information about the epidemiological characteristics of iCJD patients in Korea, we extrapolated our models based on research performed in Japan, including parameters such as the distribution of the incubation period and the cumulative incidence of iCJD $[8,18]$. Therefore, the accuracy of our predictive model may be slightly exaggerated. Second, in addition to using extrapolated models, we applied statistical methods more commonly used in the projection of acquired immunodeficiency syndrome and variant CJD data $[14,15,19]$, and we cannot statistically distinguish whether the Weibull distribution or the log-logistic distribution was more suitable for describing the latency period.Third, our models estimated the potential number of iCJD cases per year by applying the estimated incubation period after exposure to risk factors in Japan [18]. If the estimated incubation period changed for any reason, the calculated independence of the number of iCJD cases per year would be compromised. Fourth, direct sampling of the dura graft material during the study period was not possible. Fifth, surgical and medical procedures other than dura grafting that may also have resulted in iCJD, including hormone replacement therapy and corneal transplantation, were not estimated. Sixth, most of the actual cases were not confirmed by neuropathology.

In conclusion, we suggest that the occurrence of iCJD is expected to sharply decline after 2012, continuing through 2020. However, additional new cases are still expected, which necessitates a strong national surveillance system. The results indicate that the proposed model is useful for both retrospectively and prospectively estimating the size of the outbreak of iCJD in Korea.

\section{ACKNOWLEDGEMENTS}

This study was funded by the Centers for Disease Control and Prevention (KCDC), Ministry of Health and Welfare, Republic of Korea (20120224307-00). The conclusions drawn in this study represent those of the authors, not necessarily those of the KCDC. We thank the Health Insurance Review Agency for providing the health insurance dataset.

\section{CONFLICT OF INTEREST}

The authors have no conflicts of interest to declare for this study.

\section{ORCID}

Byoug-Hak Jeon https://orcid.org/0000-0001-5246-0357

Jinseob Kim https://orcid.org/0000-0002-9403-605X

Ganghyeon Kim https://orcid.org/0000-0003-2668-3412

Soochul Park https://orcid.org/0000-0002-2580-879X

SangYun Kim https://orcid.org/0000-0002-9101-5704

Hae-Kwan Cheong https://orcid.org/0000-0003-2758-9399 


\section{REFERENCES}

1. Prusiner SB. Prions. Proc Natl Acad Sci U S A 1998;95:13363-13383.

2. World Health Organization. Global surveillance, diagnosis and therapy of human transmissible spongiform encephalopathies: report of a WHO consultation, Geneva, Switzerland, 9-11 February 1998 [cited 2017 Jan 13]. Available from: http://www.who.int/csr/resources/publications/bse/WHO_EMC_ZDI 98_9/en/.

3. Kim SY, Cheong HK, An SS. Human prion diseases. J Korean Med Assoc 2008;51:1125-1138 (Korean).

4. Masters CL, Harris JO, Gajdusek DC, Gibbs CJ Jr, Bernoulli C, Asher DM. Creutzfeldt-Jakob disease: patterns of worldwide occurrence and the significance of familial and sporadic clustering. Ann Neurol 1979;5:177-188.

5. Wang J, Lewis RF. Contribution of intravestibular sensory conflict to motion sickness and dizziness in migraine disorders. J Neurophysiol 2016;116:1586-1591.

6. Duffy P, Wolf J, Collins G, DeVoe AG, Streeten B, Cowen D. Letter: possible person-to-person transmission of Creutzfeldt-Jakob disease. N Engl J Med 1974;290:692-693.

7. Will RG. Acquired prion disease: iatrogenic CJD, variant CJD, kuru. Br Med Bull 2003;66:255-265.

8. Brown P, Brandel JP, Sato T, Nakamura Y, MacKenzie J, Will RG, et al. Iatrogenic Creutzfeldt-Jakob disease, final assessment. Emerg Infect Dis 2012;18:901-907.

9. Rappaport EB. Iatrogenic Creutzfeldt-Jakob disease. Neurology 1987; 37:1520-1522.

10. Food and Drug Administration (FDA). FDA safety alert: possibly contaminated dura mater transplant material. Rockville: US Department of Health and Human Services, Public Health Service; 1987, p. $1-15$.

11. Kim HL, Do JY, Cho HJ, Jeon YC, Park SJ, Ma HI, et al. Dura mater graft-associated Creutzfeldt-Jakob disease: the first case in Korea. J Korean Med Sci 2011;26:1515-1517.

12. Triendl R. CJD link prompts ban on brain tissue use. Nature 1997;387:5.

13. Tanaka S, Fukushima M. Size of Creutzfeldt-Jakob disease epidemic associated with cadaveric dura transplantation. Neuroepidemiology 2010;34:232-237.

14. Lui KJ, Darrow WW, Rutherford GW 3rd. A model-based estimate of the mean incubation period for AIDS in homosexual men. Science 1988;240:1333-1335.

15. Kaplan ME, Schonberg SK. HIV in adolescents. Clin Perinatol 1994; 21:75-84.

16. Cox DR, Oakes D. Analysis of survival data. London: Chapman \& Hall; 1984, p. 32-45.

17. Gross AJ, Clark VA. Survival distributions: reliability applications in the biomedical sciences. New York: John Wiley; 1975, p. 112-113.

18. Hamada C, Sadaike T, Fukushima M. Projection of Creutzfeldt-Jakob disease frequency based on cadaveric dura transplantation in Japan. Neuroepidemiology 2003;22:57-64.

19. Nagatsu T. Molecular mechanisms of neurotransmission. Rinsho Shinkeigaku 2000;40:1185-1188 (Japanese).

20. National CJD Research \& Surveillance Unit (NCJDRSU). Diagnostic criteria [cited 2017 Jan 13]. Available from: http://www.cjd.ed.ac. uk/documents/criteria.pdf.

21. Hoshi K, Yoshino H, Urata J, Nakamura Y, Yanagawa H, Sato T. Creutzfeldt-Jakob disease associated with cadaveric dura mater grafts in Japan. Neurology 2000;55:718-721.

22. Centers for Disease Control and Prevention. Update: Creutzfeldt-Jakob disease associated with cadaveric dura mater grafts--Japan, 1978-2008. MMWR Morb Mortal Wkly Rep 2008;57:1152-1154.

23. Brooke FJ, Boyd A, Klug GM, Masters CL, Collins SJ. Lyodura use and the risk of iatrogenic Creutzfeldt-Jakob disease in Australia. Med J Aust 2004;180:177-181.

24. Brandel JP, Peckeu L, Haïk S. The French surveillance network of Creutzfeldt-Jakob disease. Epidemiological data in France and worldwide. Transfus Clin Biol 2013;20:395-397.

25. Brown P, Brandel JP, Preece M, Sato T. Iatrogenic Creutzfeldt-Jakob disease: the waning of an era. Neurology 2006;67:389-393.

26. Hamaguchi T, Sakai K, Noguchi-Shinohara M, Nozaki I, Takumi I, Sanjo N, et al. Insight into the frequent occurrence of dura mater graftassociated Creutzfeldt-Jakob disease in Japan. J Neurol Neurosurg Psychiatry 2013;84:1171-1175.

27. Tateishi J. Creutzfeldt-Jakob disease transmitted through cadaveric dura transplantation. J Clin Exp Med 1997;181:992-993 (Japanese).

28. Nakamura Y, Aso E, Yanagawa H. Relative risk of Creutzfeldt-Jakob disease with cadaveric dura transplantation in Japan. Neurology 1999; 53:218-220. 